\title{
Communicating information about body weight status
}

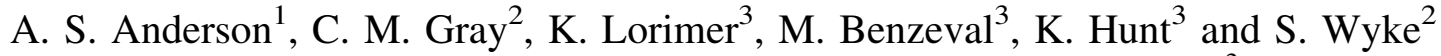 \\ ${ }^{1}$ Centre for Public Health Nutrition Research, University of Dundee, Dundee DD1 9SY, UK, ${ }^{2}$ Alliance for Self Care \\ Research, University of Stirling, Stirling FK9 4LA, UK and ${ }^{3}$ MRC Social and Public Health Sciences Unit, University of \\ Glasgow, Glasgow G12 8RZ, UK
}

Over $65 \%$ of Scottish adults are overweight or obese and current trends suggest that this figure will increase ${ }^{(1)}$. As people in general get bigger, individual perceptions of what is 'normal' stature are changing ${ }^{(2)}$. This 'normalisation' of overweight means that people may not realise that the terms 'overweight' or 'obese', and the associated risk of ill health apply to them. The societal stigmatisation associated with excess body weight and the terms used to describe it can make discussions between health professionals and their patients challenging. The current study aims to describe people's response to weight status terminology in order to inform health promotion communications about overweight/obesity action.

Participants were recruited from the longitudinal West of Scotland Twenty-07 study ${ }^{(3)}$, where they had recently learned their BMI and body fat composition via a feedback letter. In-depth, qualitative interviews (lasting 30-90 min) were undertaken with men and women (in their mid-30s and mid-50s) to investigate the knowledge of health risks associated with excess body weight and response to weight status terminology in relation to self-image and motivation to lose weight.

In total, 377 people were invited to participate, $80(21 \%)$ agreed and $50(13 \%)$ completed the interview process. When asked about health risks of overweight/obesity most spoke immediately and with confidence about the risks of CHD. Others mentioned increased risk of diabetes but far fewer spoke about the risks of cancer and were far less confident in their assertions. Most participants thought the term 'overweight' was acceptable, but few overweight or obese respondents believed that being told they were overweight would in itself motivate them to lose weight. Some interviewees (particularly younger men) preferred to refute the evidence that they were overweight themselves. Most thought the term 'obese' was negative ('offensive', 'degrading' and 'derogatory') although a number of people felt it would be a legitimate term if used by a health professional and would encourage weight loss. Concern was expressed over people's understandings of 'BMI', and many respondents favoured terms that made the links between excess weight and ill-health more explicit, e.g. 'excessively' or 'unhealthily' overweight.

In conclusion, response to weight status terminology is that fraught and health professionals should exercise sensitivity in its use to ensure that people fully understand their personal weight status and risk of ill health, and remain engaged with advice aimed at motivating a change in lifestyle.

The funding for this study was provided by Cancer Research UK (Grant No: C10877/A 15032).

1. Scottish Government (2009) The Scottish Health Survey 2008. http://www.scotland.gov.uk/Publications/2009/09/28102003/0

2. Johnson F, Cooke L, Croker H et al. (2008) Changing perceptions of weight in Great Britain: comparison of two population surveys. Br Med J 337, a494.

3. Benzeval M, Der G, Ellaway A et al. (2009) Cohort profile: West of Scotland Twenty-07 Study: health in the community. Int J Epidemiol 38, $1215-1223$. 